\title{
Correlation between pigmented arc and epithelial thickness (COPE) study in orthokeratology-treated patients using OCT measurements
}

\author{
Chun-Fu Liu ${ }^{1,2,3} \cdot$ Jiahn-Shing Lee ${ }^{2,4} \cdot$ Chi-Chin Sun $\mathbb{1}^{1,5} \cdot$ Ken-Kuo Lin ${ }^{2,4} \cdot$ Chiun-Ho Hou ${ }^{2,4} \cdot$ Ling Yeung ${ }^{1,2} \cdot$ \\ Shu-Yen Peng ${ }^{1,2}$
}

Received: 15 October 2018 / Revised: 23 June 2019 / Accepted: 12 July 2019 / Published online: 7 August 2019

(c) The Author(s), under exclusive licence to The Royal College of Ophthalmologists 2019

\begin{abstract}
Purpose To determine the intensity of corneal pigmented arc in orthokeratology (ortho-k)-treated children, and its correlation with key epithelial thickness measurements obtained by anterior segment optical coherence tomography (AS-OCT). Methods This study is a retrospective case series. Medical records of children who received ortho-k treatment for myopia control in our hospital were reviewed. Intensity of ortho-k-associated pigmented arc and its correlation with key epithelial thickness parameters in the central 7-mm-diameter zone obtained by AS-OCT was examined. The subjects were further divided into apparent and unapparent pigmented arc groups for severity comparison.

Results The mean age of children was 11.4 years, and the incidence of corneal pigmented arc was $92.2 \%$ after lens wear for a mean duration of 21.2 months. Intensity of pigmented arc was found to be significantly correlated with key epithelial thickness parameters, including maximum and minimum epithelial thickness (Spearman's rank correlation coefficient $(r s)=$ $0.404, P=0.003 ; r s=-0.426, P=0.002$, respectively), the difference between them (Min-Max) $(r s=-0.624, P<0.001)$ and standard deviation $(r s=0.659, P<0.001)$. Significant correlation between intensity of pigmented arc and ortho-k target power $(r s=0.454, P=0.001)$ was found. Comparison between the two groups showed significant difference in the same key epithelial thickness parameters.

Conclusions Children receiving ortho-k treatment tended to develop pigmented arcs. Significant correlation between intensity of pigmented arc and key epithelial thickness parameters was observed. AS-OCT can be a useful tool for predicting intensity of pigmented arc in ortho-k-treated children.
\end{abstract}

\section{Introduction}

Orthokeratology (ortho-k) with wearing customized, rigid contact lenses is a means for correcting refractive errors [1]. It has been previously established that wearing ortho-k lenses

Shu-Yen Peng, MD

stu9501407@hotmail.com

1 Department of Ophthalmology, Chang Gung Memorial Hospital, Keelung, Taiwan

2 College of Medicine, Chang Gung University, Taoyuan, Taiwan

3 Program in Molecular Medicine, National Yang Ming University, Taipei, Taiwan

4 Department of Ophthalmology, Chang Gung Memorial Hospital, Linkou, Taiwan

5 Department of Chinese Medicine, College of Medicine, Chang Gung University, Taoyuan, Taiwan can flatten the central corneal curvature to achieve temporary improvement in unaided visual acuity [2], and also slow myopia progression [3-5]. Cornea epithelial layer reshaped by ortho-k lenses has been reported [6,7], and the change was considered stable after 1-3 months of corrective lens wear overnight $[8,9]$. Although effective for controlling myopia and correcting refractive problems [2-5], ortho-k may cause complications, such as corneal pigmented arc, superficial corneal epithelial damage, and corneal ulcer [10-12].

Previous studies have indicated the occurrence of iron deposition in the epithelium under corneal conditions including Fleischer's ring, Ferry's line, and Stocker's line $[13,14]$. Pigmented arc in ortho-k-treated patients can also be taken as another condition of iron deposition in the epithelium. However, the precise etiology of pigmented arc remains largely unknown. It has been speculated that iron deposits in epithelial cells owing to pooling of tears between irregularly shaped cornea and ortho-k lens [2, 15]. This speculation is supported by the observation that 
pigmented arcs develop primarily in the reverse curve zone, where tears accumulate after overnight lens wear by ortho$k$-treated subjects $[10,15,16]$. However, design of the reverse curve varies significantly between ortho-k lens of different brands, and adjustment can hardly be made by clinicians owing to patent restriction.

In Taiwan, the number of children receiving self-paid ortho-k therapy for myopia control has been on the increase. Duration of lens wear among them is expected to be longer because myopia usually becomes stabilized after adulthood [17]. The positive correlation between duration of lens wear and both incidence and intensity pigmented arc merits attention and caution [15]. However, related research on the long-term safety of ortho-k-associated pigmented arc in children remains scarce. A search on Pubmed database revealed all pigmented arc studies having follow-up within 15 months after lens wear, most of the subjects being adults or teenagers [2, 10-12, 15], and only one case report addressing the disappearance of pigmented arc in two adult patients after termination of 1-year ortho-k treatment [18]. Long-term influence of ortho-k-associated pigmented arc on the cornea is still unknown. To further improve the safety of ortho-k treatment, efforts should be made to prevent arc development, especially among ortho-k-treated children.

Anterior segment optical coherence tomography (AS-OCT) has been shown to accurately measure corneal epithelial thickness of subjects after ortho-k lens wear [7, 19-22]. This technique can provide three-dimensional measurements of the corneal epithelial structure up to $9 \mathrm{~mm}$ in diameter with a new wide-field pachymetry scan pattern. Direct epithelial analysis makes possible the determination of crucial parameters for ortho-k lens design, which may be associated with intensity of pigmented arc. Understanding such association would enable the design of customized pigmented-arc-free ortho-k lenses.

This study hypothesizes that intensity of pigmented arc is associated with corneal epithelial thickness. AS-OCT was performed under new wide-field pachymetry scan to obtain key epithelial thickness measurements in the central 7-mm-diameter zone for determining their correlation with pigmented arc intensity in ortho-k-treated children $[8,9]$.

\section{Materials and methods}

\section{Subjects}

The current study is a retrospective case series. Medical records of patients undergoing ortho-k treatment for myopia control between January 2015 and February 2018 at Keelung Chang Gung Memorial Hospital were reviewed. The study was approved by the Institutional Review
Board of Chang Gung Memorial Hospital (approval no. 201800215b0) and followed the tenets of the Declaration of Helsinki.

\section{Exclusion criteria}

Patients who (1) wore their ortho-k lenses overnight for $<90 \%$ of the follow-up period, (2) developed complications during ortho-k treatment, (3) discontinued lens wear for $>1$ week, (4) were lost to follow-up in $<6$ months after initiation of ortho-k treatment, (5) did not receive AS-OCT at 6 months after initiation of ortho-k treatment, and (6) wore ortho-k lenses of brands other than DreimLens (Brighten Optix Co., Taiwan) were excluded from the study.

\section{Ocular examination}

All patients received standard scheduled ocular examinations before and after initiation of ortho-k treatment. Pre-treatment tests included best-corrected visual acuity, cycloplegic refraction power, keratometry (Auto Ref/ Keratometer ARK-1a/ARK-1; Nidek Co., Ltd., Gamagori, Aichi, Japan), corneal topography (Orbscan IIz, Bausch \& Lomb, Rochester, New York, USA), external eye photography and axial length measurement (IOLMaster 500, Carl Zeiss Meditec AG, Jena, Germany). Follow-up examinations after initiation of ortho-k treatment included best-corrected visual acuity, uncorrected visual acuity, slit-lamp biomicroscopy (BQ 900, Haag-Streit AG, Koeniz, Switzerland) before and after fluorescence staining of the cornea at 1 week, 1 month, and every 3 months thereafter, as well as axial length measurement every 6 months. From May 2017, AS-OCT under new wide-field pachymetry scan (Optovue RTVue XR Avanti, Optovue Inc., Fremont, CA, USA) was performed on subjects after lens wear for at least 6 months and thereafter as necessary. AS-OCT images of the best quality were selected for analysis. Criteria for selection included good pupil-cantered scan, minimal eyelash, and eyelid disturbance.

\section{Ortho-k lenses}

All subjects wore ortho-k lenses of the same brand (DreimLens; Brighten Optix Co., Taiwan). Astigmatic lenses were also prescribed for better centration as necessary. Ortho-k lenses used in the current study were of a reversegeometry design (Boston XO material by Brighten Optix Co., Taiwan) with overall diameters ranging between 10.2 and $10.8 \mathrm{~mm}$ and back optic zone diameter of $6 \mathrm{~mm}$. All prescriptions were prescribed by the same ophthalmologist (C-F, Liu). 


\section{Optical coherence tomography}

This study used a Fourier-domain optical coherence tomography system with a corneal adaptor module. The cornea was imaged using a high-magnification adaptor lens focused at the pupil center with a new 9-mm wide-field pachymetry scan (PachymetryWide scan, eight radial lines with 9-mm scan length, 1056 axial scans each radial line, repeated four times) and corneal power data were obtained with a corneal power scan (Pachymetry + Cpwr scan, eight radial lines with 6-mm scan length, 1020 axial scans each radial line, repeated five times).

Epithelial thickness was measured as the distance between the air-tear interface (first curve) and the epithelium-Bowman's layer boundary (second curve). All ASOCT images were processed and verified according to the manufacturer's guidelines by adjusting the first and second curves to fit the real corneal contour. A 9-mm-diameter wide epithelial thickness map (wETM) was generated from the wide-field pachymetry scan data obtained and was employed to verify that all scanning lines matched the real contour of different layers with no sign of central epithelial islands.

Key epithelial thickness parameters in the 7-mmdiameter zone including average epithelial thickness of superior and inferior zones, minimum, and maximum epithelial thickness, the difference between them (Min-Max), and standard deviation acquired under wide-field pachymetry scan were further analyzed. Anterior and posterior corneal powers in the central 3-mm-diameter zone were also measured and only corneal power scans taken at the same OCT exam with "Good" measurement reliability rating were analyzed. To illustrate data obtained and analyzed, Fig. 1 shows examples of images and measurements obtained from the right eye of a 14-year-old female subject after 29 months of ortho-k treatment using AS-OCT new wide-field pachymetry and corneal power scan. Figure 2 is the right-eye wETM of the same subject for more detailed illustration of key epithelium statistics in the central 7-mmdiameter zone.

\section{Pigmented arc grading}

Pigmented arcs were directly graded by the same ophthalmologist (C-F, Liu) at each patient visit using a slit lamp with narrow decentered slit beam and wide-beam blue light according to the scale used by Cho et al. [15]. In brief, grade 0 represents no detectable pigmented arc; grade 1, arcs detectable under white light, but not visible with blue light; grade 2 , arcs easily observed under both white and blue light but have ill-defined margins; and grade 3 , arcs very easily observed under both white and blue light and have well-defined margins. The patients were further divided into two groups according to severity of pigmented arcs: apparent (grades 2 and 3, blue-light detectable) and unapparent pigmented arc (grades 0 and 1, blue-light undetectable) groups for further analysis.

\section{Statistical analysis}

Standard deviation in epithelial thickness of apparent and unapparent pigmented arc groups was assumed to be 5 and $4 \mu \mathrm{m}$, respectively; with a common pooled standard deviation of 1 . Given alpha level of $5 \%$ and power of $90 \%$, the required minimum sample size was 44 .

All data were analyzed using SPSS software version 20.0 (SPSS, Inc., Chicago, IL, USA). Intensity of pigmented arc after lens wear and its correlation with key epithelial thickness parameters was determined using bivariate Spearman's Rho correlation test. Statistically significant differences were defined as $p<0.05$ (two-tailed). As for the comparison between apparent and unapparent pigmented arc groups, Chi-square analysis was applied to categorical data and an independent samples $t$ test was applied to continuous data. Fisher's exact test was used when one or more of the cells in the $\mathrm{X}^{2}$ analysis had expected values below 5 ; statistically significant differences were defined as $p<0.05$.

\section{Results}

Medical records of 77 eyes from 40 children were reviewed. Twelve eyes from six cases were excluded because they were lost to follow-up in $<6$ months after initiation of ortho-k treatment; 11 eyes from six cases were excluded owing to overnight ortho-k lens wear for $<90 \%$ of the follow-up period; 1 eye was excluded owing to a suspected infection; and 2 eyes from one case were excluded owing to AS-OCT data missing. A total of 51 eyes from 26 patients (age range, 9-15 years) were included in the final analysis.

Table 1 summarizes the demographic and ortho-k treatment data of the studied patients. Of note is that pigmented arcs were always observed in the reverse curve zone (Fig. 1a).

Table 2 summarizes the correlation of pigmented arc intensity with epithelial thickness, corneal power, and ortho-k treatment parameters. As can be seen, intensity of pigmented arc had significant correlation with maximum and minimum epithelial thickness, Min-Max value, and standard deviation. Moreover, significant positive correlation between intensity of pigmented arc and ortho-k target power was also found $(P=0.001)$; and there is a trend that the longer the ortho-k lens wear duration, the greater the intensity of pigmented arcs became $(P=0.071)$. On the contrary, no significant correlation between compression 
Fig. 1 Right eye of a 14-yearold girl under ortho-k treatment for 29 months. a Corneal pigmented arc observed. b Wide epithelial thickness map (wETM) for verifying that all scanning lines match the real contour of different layers with no sign of central epithelial islands. c Vertical line-scan showing pigmented epithelium at inferior mid-peripheral cornea (arrowhead). d Corneal power measurements obtained by ASOCT with "Good" measurement reliability rating. e Epithelium statistics in central 7-mmdiameter zone obtained using AS-OCT under wide-field pachymetry scan
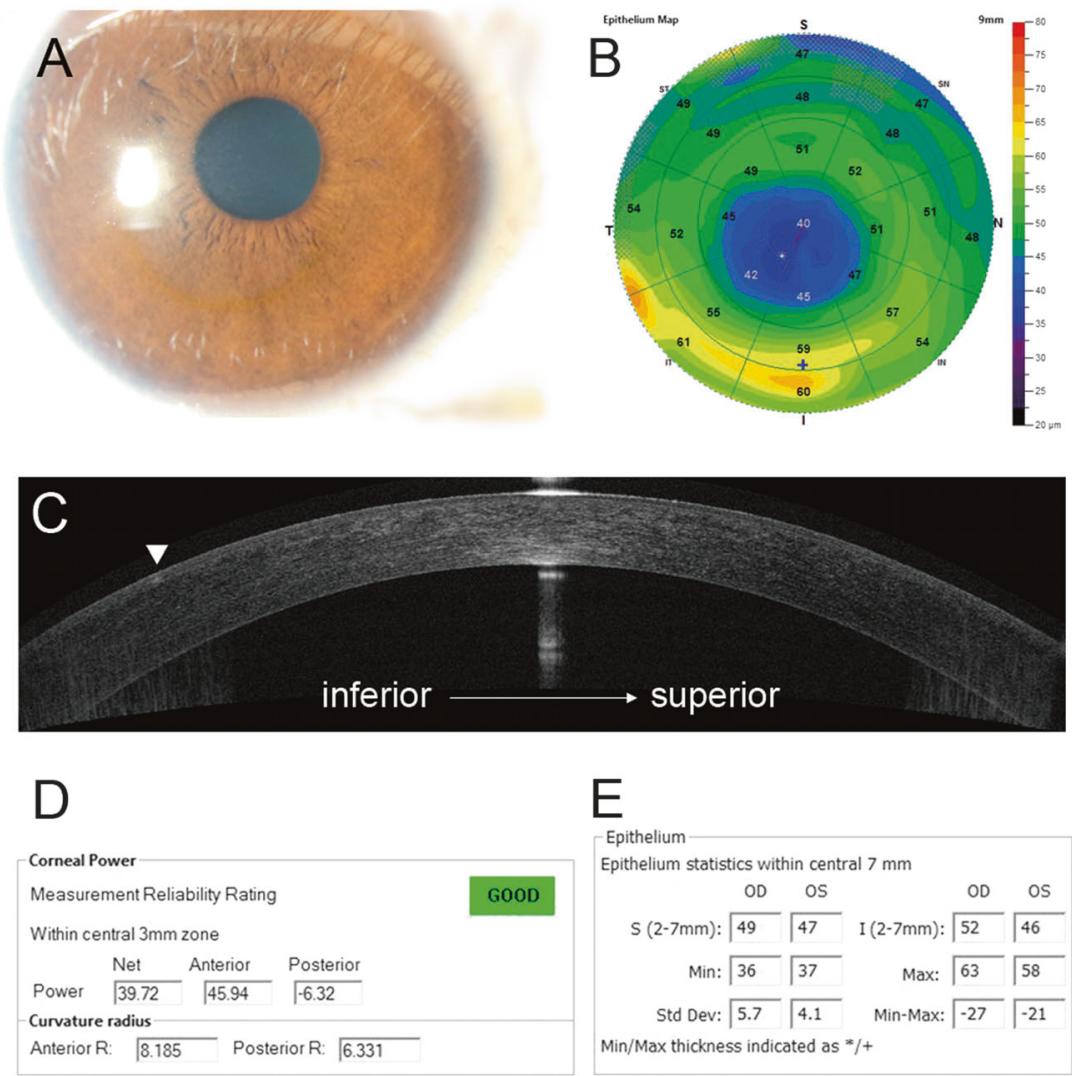

effect as a result of average alignment curve power minus average corneal curve power and intensity of pigmented arc $(P=0.237)$ was observed. Furthermore, intensity of pigmented arc showed significant correlation only with posterior corneal power in the central 3-mm-diameter zone and all corneal power scan had "Good" measurement reliability rating.

Table 3 shows comparison between the apparent and unapparent pigmented arc groups. As can be seen, there are significant differences in the same key epithelial thickness parameters for similar ortho-k lens wear duration but no significant difference in all corneal power measurements.

\section{Discussion}

To the best of our knowledge, the current study is the first to describe a correlation between intensity of pigmented arc and corneal epithelial thickness in school-aged children (9-15 years old) wearing ortho-k lenses. It is also the first to use AS-OCT new wide-field pachymetry scan to analyze corneal epithelial thickness during ortho-k treatment. Results of AS-OCT revealed that intensity of pigmented arc increased with lower minimum and higher maximum epithelial thickness, more negative Min-Max value and larger standard deviation in the central 7-mm-diameter zone as well as higher target power of ortho-k prescription (Tables 2, 3).

Among the key epithelial thickness parameters, standard deviation and Min-Max value showed the greatest correlation with intensity of pigmented arc $(P<0.001)$, indicating their significant impact on arc development. At higher target power prescription, the Min-Max value becomes more negative while the standard deviation becomes larger, implying greater curvature change along the base curve to reverse curve. Whether changing reverse curve design would decrease intensity of pigmented arc merits further investigation.

The insignificant correlation between intensity of pigmented arc and average epithelial thickness of superior and inferior zones can be attributed to opposite changes in thickness of the two zones offset by the respective changes in base curve and reverse curve of ortho-k lens.

Evidence supporting myopia control through ortho-k lens wear has been increasing and this treatment has been popularly used among the school-age around the world. With AS-OCT, three-dimensional information of epithelial structure in zones up to $9 \mathrm{~mm}$ in diameter can be obtained, thus facilitating the design of customized and safe ortho-k treatment with least or no development of pigmented arcs.

In line with previous results $[15,16]$, significant positive correlation between intensity of pigmented arc and target 


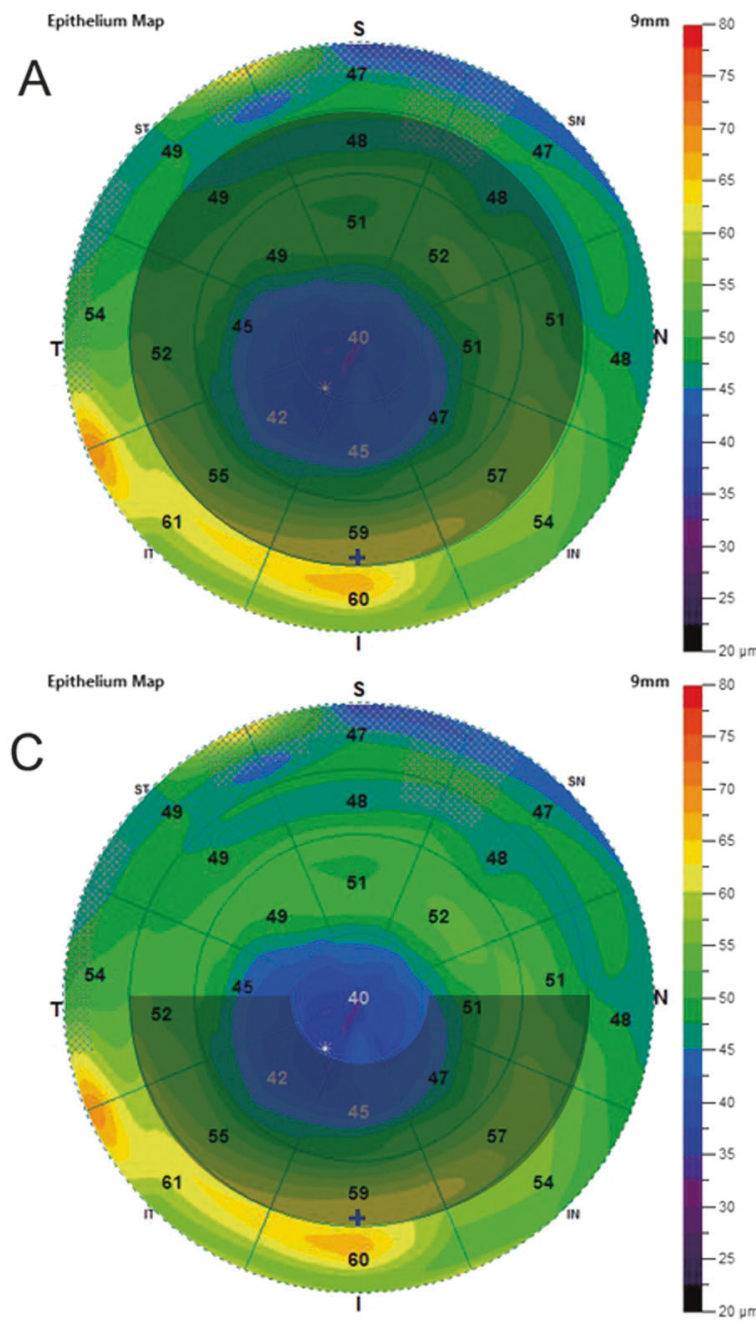

Fig. 2 Right-eye wide epithelial thickness map of a 14-year-old girl under ortho-k treatment for 29 months. a Epithelium statistics in central 7-mm-diameter zone. b Average epithelial thickness in superior

Table 1 Demographic and ortho-k treatment data of studied patients

\begin{tabular}{ll}
\hline & Cases $(n=51)$ \\
\hline Age (years) & $11.4 \pm 2.0$ \\
Gender (male: female) & $16: 10$ \\
Target power (D) & $-3.48 \pm 1.33$ \\
Diameter (mm) & $10.55 \pm 0.15$ \\
Alignment curve ${ }^{\mathrm{a}}(\mathrm{D})$ & $42.57 \pm 1.13$ \\
Astigmatism (eye) & Yes $28:$ No 23 \\
Mean AS-OCT exam time point (months) & $19.6 \pm 7.4$ \\
Lens wear duration (months) & $21.2 \pm 6.7$ \\
Incidence of pigmented arc $(\%)$ & 92.2 \\
\hline
\end{tabular}

Continuous data are presented as mean \pm standard deviation

AS-OCT anterior segment optical coherent tomography, D Dioptre, Ortho-k orthokeratology

${ }^{a}$ Astigmatism prescription using average alignment curve for calculation

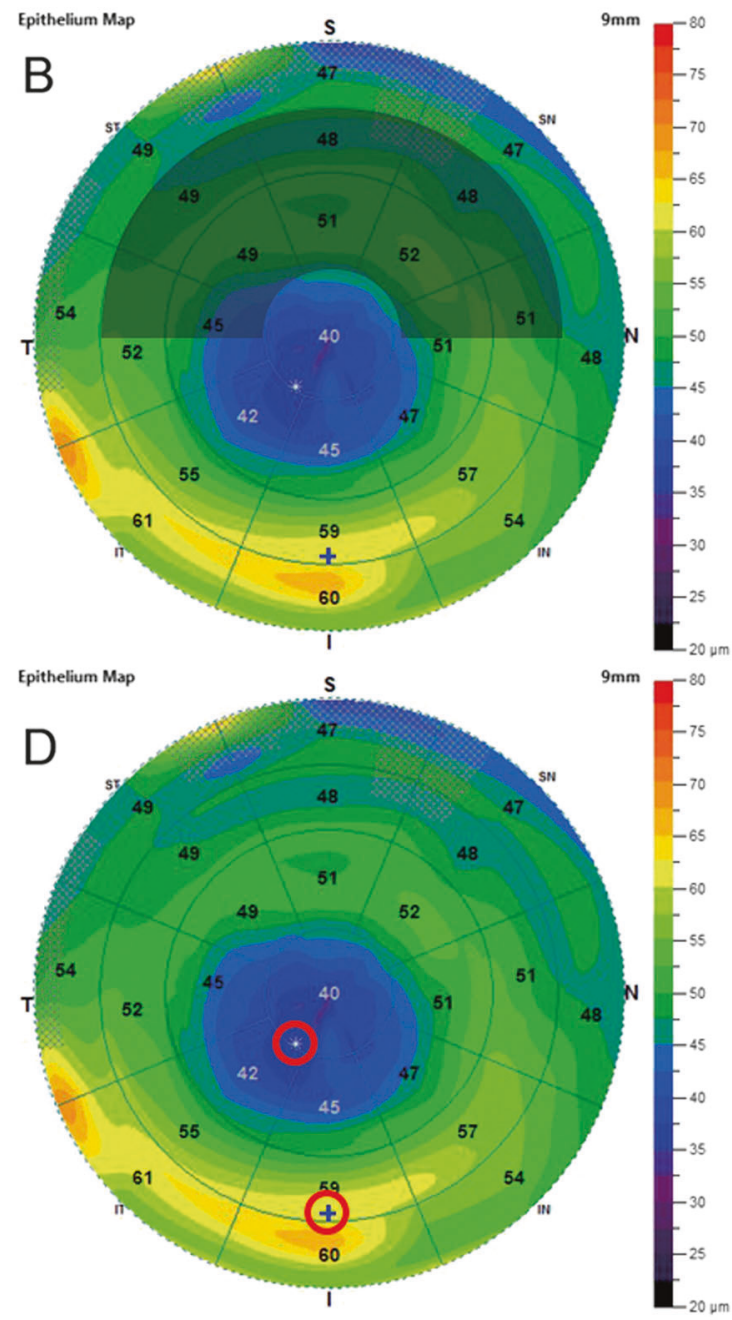

2-7-mm-diameter zone. c Average epithelial thickness in inferior 2-7mm-diameter zone. d " + " marks point of maximum epithelial thickness and "** marks point of minimum epithelial thickness

power of ortho-k prescription was observed in the present study. In current medical practice, reverse curve design of the same target power may vary in height, curve, shape, and width in ortho-k lenses of different brands. However, it remains unclear whether the incidence and intensity of pigmented arc may also differ among ortho-k lenses of the same target power but of different designs and brands. Knowledge of such would contribute to the development of pigmented arc-free ortho-k treatment.

It is known that ortho-k treatment can reshape the cornea by changing its epithelial layer [6, 20], and variations in epithelial thickness are mainly associated with changes along the base curve to the reverse curve of ortho-k lens. The present statistical results and location of pigmented arcs both highlight the significance of the reverse curve in development of pigmented arcs. In other words, changing the design of the reverse curve alter the key epithelial thickness 
Table 2 Correlation of pigmented arc intensity with epithelial thickness, corneal power and ortho-k treatment parameters

\begin{tabular}{lrrc}
\hline & Mean \pm SD & \multicolumn{1}{l}{$r s$} & $P$ value \\
\hline AS-OCT-measured epithelial thickness & & \\
Maximum $(\mu \mathrm{m})$ & $63.2 \pm 6.5$ & 0.404 & $0.003^{*}$ \\
Minimum $(\mu \mathrm{m})$ & $39.9 \pm 3.9$ & -0.426 & $0.002^{*}$ \\
Min-Max $(\mu \mathrm{m})$ & $-23.3 \pm 7.4$ & -0.624 & $<0.001^{*}$ \\
Standard deviation $(\mu \mathrm{m})$ & $5.5 \pm 2.0$ & 0.659 & $<0.001^{*}$ \\
Average of superior zone $(\mu \mathrm{m})$ & $52.0 \pm 4.4$ & 0.195 & 0.171 \\
Average of inferior zone $(\mu \mathrm{m})$ & $54.7 \pm 5.4$ & 0.173 & 0.225 \\
AS-OCT-measured corneal power & & & \\
Anterior (D) & $45.18 \pm 1.65$ & -0.230 & 0.104 \\
Posterior (D) & $-6.05 \pm 0.19$ & -0.314 & $0.025^{*}$ \\
Net (D) & $39.24 \pm 1.61$ & -0.262 & 0.063 \\
Ortho-k treatment parameters & & & \\
Target power (D) & $-3.48 \pm 1.33$ & 0.454 & $0.001^{*}$ \\
Diameter (mm) & $10.55 \pm 0.15$ & 0.011 & 0.941 \\
Alignment curve (D) & $42.57 \pm 1.13$ & 0.152 & 0.288 \\
Compression ${ }^{\mathrm{a}}(\mathrm{D})$ & $-0.24 \pm 0.33$ & -0.169 & 0.237 \\
Lens wear duration (months) & $21.2 \pm 6.7$ & 0.255 & 0.071 \\
\hline
\end{tabular}

$A S$-OCT anterior segment optical coherent tomography, $D$ dioptre, Min-Max minimal epithelial thickness minus maximal epithelial thickness, Ortho- $k$ orthokeratology, SD Standard deviation, $r s$ Spearman's rank correlation coefficient

" $P$ values $<0.05$ calculated by Spearman's correlation analysis

${ }^{a}$ Compression: average alignment curve power minus average corneal curve power

parameters, which may in turn affect the intensity of pigmented arc. Nevertheless, the range of alterations to be made without sacrificing the purpose for myopia control and refraction error correction warrants further investigation.

The current finding of $92.2 \%$ incidence of pigmented arc after a mean lens wear duration of 21.2 months is in line with previous results of over $90 \%$ incidence after 12 months of lens wear $[15,16]$. While Cho et al. reported significant positive correlation of incidence and intensity of pigmented arc with duration of lens wear [15], this study observed only positive correlation between intensity of pigmented arc and duration of lens wear $(P=0.071)$. The discrepancy in findings may be due to variations in lens wear duration in subjects having different grades of pigmented arcs and the retrospective cross-section design of this study. Although longer ortho-k lens wear would lead to higher pigmented arc intensity, other key epithelial thickness parameters and target power are also influencing factors. Large-scale long-term prospective study is required for further clarification on the above correlation.

Prior research reports demonstrated no change in posterior corneal curvature as a result of ortho-k treatment $[8,23]$. However, corneal power measurements show significant positive correlation between posterior corneal
Table 3 Comparison of data between apparent and unapparent pigmented arc groups

\begin{tabular}{|c|c|c|c|}
\hline Pigmented arc & $\begin{array}{l}\text { Apparent } \\
(n=38)\end{array}$ & $\begin{array}{l}\text { Unapparent } \\
(n=13)\end{array}$ & $P$ value \\
\hline Age (years) & $11.4 \pm 2.1$ & $11.5 \pm 1.6$ & 0.950 \\
\hline Gender (male: female) & 27: 11 & $5: 8$ & $0.050^{\mathrm{a}}$ \\
\hline $\begin{array}{l}\text { Mean AS-OCT exam time point } \\
\text { (months) }\end{array}$ & $20.3 \pm 7.3$ & $17.8 \pm 7.4$ & 0.296 \\
\hline \multicolumn{4}{|c|}{ AS-OCT-measured epithelial thickness } \\
\hline Maximum $(\mu \mathrm{m})$ & $64.4 \pm 6.3$ & $59.6 \pm 6.0$ & $0.019 *$ \\
\hline Minimum $(\mu \mathrm{m})$ & $39.1 \pm 3.4$ & $42.3 \pm 4.4$ & 0.008 \\
\hline $\operatorname{Min}-\operatorname{Max}(\mu \mathrm{m})$ & $-25.4 \pm 7.1$ & $-17.3 \pm 4.5$ & $<0.001 *$ \\
\hline Standard deviation $(\mu \mathrm{m})$ & $6.1 \pm 1.9$ & $3.7 \pm 0.8$ & $<0.001 *$ \\
\hline Average of superior zone $(\mu \mathrm{m})$ & $52.3 \pm 4.0$ & $51.2 \pm 5.6$ & 0.510 \\
\hline Average of inferior zone $(\mu \mathrm{m})$ & $55.2 \pm 4.8$ & $53.0 \pm 6.8$ & 0.287 \\
\hline \multicolumn{4}{|l|}{ AS-OCT-measured corneal power } \\
\hline Anterior (D) & $45.03 \pm 1.67$ & $45.62 \pm 1.57$ & 0.272 \\
\hline Posterior (D) & $-6.06 \pm 0.20$ & $-6.00 \pm 0.4$ & 0.279 \\
\hline Net (D) & $39.07 \pm 1.64$ & $39.72 \pm 1.50$ & 0.212 \\
\hline \multicolumn{4}{|l|}{ Ortho-k treatment parameters } \\
\hline Target power (D) & $3.74 \pm 1.32$ & $2.73 \pm 1.04$ & $0.009^{*}$ \\
\hline Diameter (mm) & $10.55 \pm 0.15$ & $10.58 \pm 0.16$ & 0.510 \\
\hline Alignment curve $^{c}$ (D) & $42.67 \pm 1.12$ & $42.27 \pm 1.13$ & 0.267 \\
\hline Compression $^{\mathrm{b}}(\mathrm{D})$ & $-0.27 \pm 0.31$ & $-0.18 \pm 0.38$ & 0.433 \\
\hline Astigmatism (Eye) & 22: 16 & 6: 7 & $0.463^{\mathrm{c}}$ \\
\hline Lens wear duration (months) & $21.9 \pm 6.7$ & $19.0 \pm 6.4$ & 0.179 \\
\hline
\end{tabular}

Continuous data are presented as mean \pm standard deviation

$A S$-OCT anterior segment optical coherent tomography, $D$ dioptre, Min-Max minimal epithelial thickness minus maximal epithelial thickness, Ortho- $k$ orthokeratology

${ }^{\mathrm{a}}$ Fisher's Exact test

${ }^{\mathrm{b}}$ Compression: average alignment curve power minus average corneal curve power

${ }^{\mathrm{c}}$ Pearson Chi-square

*P values $<0.05$ calculated by independent $t$ test

curvature and intensity of pigmented arc after ortho-k lens wear, indicating the possible influence of initial corneal condition on intensity of pigmented arc. On the other hand, this observation could be a measurement error owing to increasing signal disturbance and reflectance from the epithelium compressed by the base curve in the central corneal zone. Measurement error is likely the reason behind such observation as echoed by no significant difference found between the two groups with different severity of pigmented arcs. Whether changes in posterior corneal curvature could be owing to ortho-k lens compression should also be confirmed by AS-OCT in future studies.

Limitations of the present study included small number of cases, the retrospective and cross-section methodology, the lack of baseline AS-OCT data for comparing changes before and after initiation of ortho-k treatment, and the absence of a group wearing ortho-k lens of different brands for comparison. Safety is always the first priority in ortho-k therapy; hence, all ortho-k-treated patients should receive 
detailed ocular examinations before and after lens wear with a standard follow-up schedule. Details of these examinations may compensate data loss and examination discrepancies of the retrospective study. Nevertheless, to understand better the correlation between epithelial changes after initiation of ortho-k treatment would merit a prospective long-term longitudinal study with the above limitations addressed.

In summary, this study found that incidence of ortho-kassociated pigmented arc in school-age children was $92.2 \%$ after lens wear for a mean of 21.2 months; and the intensity of pigmented arc was significantly correlated with target power of ortho-k prescription and key epithelial thickness measurements in the central 7-mmdiameter zone obtained by AS-OCT under wide-field pachymetry scan. Hence, AS-OCT, when performed promptly after stabilization of anterior corneal curvature, could be a useful tool for predicting intensity of pigmented arc in ortho-k-treated young patients $[6,8,9]$. The three-dimensional epithelial structural information provided by AS-OCT would facilitate the design of customized pigmented arc-free ortho-k treatment.

\section{Summary}

\section{What was known before}

- Children receiving orthokeratology (ortho-k) treatment tended to develop pigmented arcs.

- Intensity of pigmented arc was significantly correlated with ortho-k target power and change in corneal apical radius.

\section{What this study adds}

- Significant correlation between intensity of pigmented arc and key epithelial thickness parameters were observed.

- Anterior segment optical coherence tomography can be a useful tool for predicting intensity of pigmented arc in ortho-k-treated children.

Acknowledgements We acknowledge the financial support of the Maintenance Project of the Bio-statistical Consultation Center under Grant CLRPG2G0082 and thank the technicians of the Department of Ophthalmology, Chang Gung Memorial Hospital, Keelung for their technical assistance.

\section{Compliance with ethical standards}

Conflict of interest The authors declare that they have no conflict of interest.
Publisher's note: Springer Nature remains neutral with regard to jurisdictional claims in published maps and institutional affiliations.

\section{References}

1. Liu YM, Xie P. The safety of orthokeratology-a systematic review. Eye Contact Lens. 2016;42:35-42.

2. Hiraoka T, Furuya A, Matsumoto Y, Okamoto F, Kakita T, Oshika T. Corneal iron ring formation associated with overnight orthokeratology. Cornea. 2004;23:S78-81.

3. Li X, Friedman IB, Medow NB, Zhang C. Update on orthokeratology in managing progressive myopia in children: efficacy, mechanisms, and concerns. J Pedia Ophthalmol Strabismus. 2017;54:142-8.

4. Chen C, Cheung SW, Cho P. Myopia control using toric orthokeratology (TO-SEE study). Invest Ophthalmol Vis Sci. 2013;54:6510-7.

5. Cho P, Cheung SW. Retardation of myopia in Orthokeratology (ROMIO) study: a 2-year randomized clinical trial. Invest Ophthalmol Vis Sci. 2012;53:7077-85.

6. Swarbrick HA, Wong G, O'Leary DJ. Corneal response to orthokeratology. Optom Vis Sci. 1998;75:791-9.

7. Kim WK, Kim BJ, Ryu IH, Kim JK, Kim SW. Corneal epithelial and stromal thickness changes in myopic orthokeratology and their relationship with refractive change. PLoS ONE. 2018;13: $\mathrm{e} 0203652$.

8. Chen D, Lam AK, Cho P. Posterior corneal curvature change and recovery after 6 months of overnight orthokeratology treatment. Ophthalmic Physiol Opt. 2010;30:274-80.

9. Tsukiyama J, Miyamoto Y, Higaki S, Fukuda M, Shimomura Y. Changes in the anterior and posterior radii of the corneal curvature and anterior chamber depth by orthokeratology. Eye Contact Lens. 2008;34:17-20.

10. Liang JB, Chou PI, Wu R, Lee YM. Corneal iron ring associated with orthokeratology. J Cataract Refract Surg. 2003;29:624-6.

11. Cho P, Chui WS, Mountford J, Cheung SW. Corneal iron ring associated with orthokeratology lens wear. Optom Vis Sci. 2002;79:565-8.

12. Gonzalez-Meijome JM, Gonzalez-Perez J, Garcia-Porta N, DiazRey A, Parafita-Mato MA. Pigmented corneal ring associated with orthokeratology in Caucasians: case reports. Clin Exp Optom. 2012;95:548-52.

13. Barraquer-Somers E, Chan CC, Green WR. Corneal epithelial iron deposition. Ophthalmology. 1983;90:729-34.

14. Hiratsuka Y, Nakayasu K, Kanai A. Secondary keratoconus with corneal epithelial iron ring similar to Fleischer's ring. Jpn J Ophthalmol. 2000;44:381-6.

15. Cho P, Cheung SW, Mountford J, Chui WS. Incidence of corneal pigmented arc and factors associated with its appearance in orthokeratology. Ophthalmic Physiol Opt. 2005;25:478-84.

16. Charm J, Cho P. High myopia-partial reduction ortho-k: a 2-year randomized study. Optom Vis Sci. 2013;90:530-9.

17. Hou W, Norton TT, Hyman L, Gwiazda J, Group C. Axial elongation in myopic children and its association with myopia progression in the correction of myopia evaluation trial. Eye Contact Lens. 2018;44:248-59.

18. Cho P, Chui WS, Cheung SW. Reversibility of corneal pigmented arc associated with orthokeratology. Optom Vis Sci. 2003;80:791-5.

19. Kim E, Ehrmann K. Assessment of accuracy and repeatability of anterior segment optical coherence tomography and reproducibility of measurements using a customised software program. Clin Exp Optom. 2012;95:432-41. 
20. Li Y, Tan O, Brass R, Weiss JL, Huang D. Corneal epithelial thickness mapping by Fourier-domain optical coherence tomography in normal and keratoconic eyes. Ophthalmology. 2012;119:2425-33.

21. Li Y, Tang M, Zhang X, Salaroli CH, Ramos JL, Huang D. Pachymetric mapping with Fourier-domain optical coherence tomography. J Cataract Refract Surg. 2010;36:826-31.
22. Qian Y, Xue F, Huang J, Qu X, Zhou X, Lanen-Wanek DV. Pachymetry map of corneal epithelium in children wearing orthokeratology contact lenses. Curr Eye Res. 2014;39:263-70.

23. Yoon JH, Swarbrick HA. Posterior corneal shape changes in myopic overnight orthokeratology. Optom Vis Sci. 2013; 90:196-204. 